\title{
Evaluation of Strategies in the Management of Infective Aortic Valve Endocarditis at German Cardiac Surgical Departments
}

\author{
Payam Akhyari1@ Hug Aubin ${ }^{1}$ Maximilian Lühr ${ }^{2}$ Ardawan Julian Rastan ${ }^{3}$ Andreas Beckmann ${ }^{4}$ \\ Martin Misfeld ${ }^{5}$ on behalf of the Working Group Aortic Valve Surgery and the Board of Directors of the \\ German Society for Thoracic Cardiovascular Surgery
}

${ }^{1}$ Department of Cardiovascular Surgery, Medical Faculty, Heinrich Heine University, Düsseldorf, Germany

2 Department of Cardiac Surgery, University Hospital, LMU Munich, Germany

${ }^{3}$ Department of Cardiac and Vascular Surgery, Herz-Kreislauf-

Zentrum Rotenburg, Rotenburg a.d. Fulda, Germany

${ }^{4}$ Deutsche Gesellschaft für Thorax-, Herz- und Gefäßchirurgie [DGTHG], Berlin, Germany

${ }^{5}$ Department of Cardiac Surgery, Heart Centre, University of Leipzig, Leipzig, Germany

Thorac Cardiovasc Surg 2019;67:624-630.

Address for correspondence Univ.-Prof. Dr. med. Payam Akhyari, Department of Cardiovascular Surgery, Medical Faculty, Heinrich Heine University, Moorenstr. 5, Düsseldorf 40225, Germany (e-mail: payam.akhyari@med.uni-duesseldorf.de).

\begin{abstract}
Keywords

- questionnaire

- infective endocarditis

- aortic valve

- common practice

- quality management
\end{abstract}

Background Surgical treatment of infective aortic valve endocarditis (AVE) remains a therapeutic challenge, necessitating interdisciplinary approach to limit morbidity and mortality in this high-risk cohort. With a considerable spectrum of available laboratory testings, imaging techniques, as well as operative strategies developed in recent years, there is a lack of standardization across cardiac surgical departments in Germany. Thus, the optimal treatment strategy of AVE has yet to be defined.

Methods A nationwide survey on infective AVE was conducted, including 64 cardiac surgical departments responding to a 41 -item questionnaire. The evaluation included common surgical practice, routine diagnostic steps, surgical techniques, perioperative medical treatment, as well as postoperative management.

Results Remarkable differences were observed among the participating institutions, including the following components of the treatment: (1) standardization and extent of the use of imaging techniques and (2) success rate in identification AVE-causing germs, (3) timing of operation in case of cerebral embolization, and (4) choice of valve prosthesis for aortic valve replacement for infective AVE.

Conclusion The findings of this survey underline the need for a nationwide registry to further elucidate the nature and course of AVE in Germany, as well as to serve as a solid basis for prospective trials, addressing the most important clinical purposes in the diagnosis and treatment of AVE. received

January 10, 2018

accepted after revision

August 23, 2018

published online

November 28, 2018 (c) 2019 Georg Thieme Verlag KG

Stuttgart · New York
Dol https://doi.org/

10.1055/s-0038-1673634. ISSN 0171-6425. 


\section{Introduction}

Patients suffering from infective aortic valve endocarditis (AVE) remain a diagnostic and therapeutic challenge. Despite considerable improvements in laboratory testing and imaging tools used for the early diagnosis, and irrespective of further development of antibiotic agents with a broader spectrum and greater potency, the early, mid- and longterm outcome of patients affected by AVE is yet limited.

Surgical therapy aiming the removal of all infected tissue is often the only curative option. To standardize and to improve clinical practice, specific recommendations have been released by international scientific societies, including guidelines for the diagnosis and management of infective endocarditis. ${ }^{1-3}$ In 2008 , a paradigm change was implied by the guidelines, restricting the use of antibiotic endocarditis prophylaxis to high-risk patients undergoing invasive procedures that are associated with a high risk of endocarditis. ${ }^{4,5}$ Since then controversies remain on the efficacy and appropriateness of the anti-infective therapy regimen recommended by the guidelines, and the updated 2015 guidelines have essentially maintained the principles of the preceding version. ${ }^{2,5}$ Among the community for heart medicine, concerns have arisen to whether the current guidelines may have led to a change in the pattern of infective endocarditis (IE) as seen in the daily practice. Moreover, considering the aforementioned debate, the adherence to the guidelines has become a matter of concern.

In 2015, the Working Group on Aortic Valve Surgery has performed a nationwide survey involving German cardiac surgical institutions to elucidate penetration and adherence of the current practice for treatment of AVE. This survey was intended to elucidate regional differences in the incidence and pattern of AVE.

\section{Methods}

In 2015, all German institutions performing cardiac surgical procedures were contacted on behalf of the Working Group on Aortic Valve Surgery of the German Society for Thoracic and Cardiovascular Surgery (DGTHG, Deutsche Gesellschaft für Thorax-, Herz-und Gefäßchirurgie, www.dgthg.de) and asked to participate in a nationwide survey. While designing the questionnaire and preparing the administrative part of the survey, necessary diligence was given to the principle of anonymous participation. A web-based questionnaire consisting of 41 items was designed by the members of the Working Group for Aortic Valve Surgery to allow for online completion (see Supplementary Data [online only] file for complete questionnaire in its original form). The various contents were presented and discussed in preceding meetings involving all members of the working group as well as selected members of the society. The first call for participation was sent during the second half of the year 2015. After initiating one memorization, the survey was closed in October 2015, providing the response of 64 centers. All responses were collected and included into a central database allowing for statistical analysis of the entire data. In a further approach, the results were regionally categorized to evaluate local differences in Germany, according to the following definition of regions:

1. Northeast: Mecklenburg-West Pomerania, Berlin, Brandenburg;

2. Northwest: Schleswig-Holstein, Hamburg, Bremen, Lower Saxony;

3. Central East: Saxony, Saxony-Anhalt, Thuringia;

4. Central West: North Rhine-Westphalia, Hessen, Saarland, Rhineland-Palatinate;

5. South: Bavaria, Baden-Wuerttemberg.

After analyzing the results, a summary of the survey results was presented at the 45th annual meeting of the German Society for Thoracic and Cardiovascular Surgery in Leipzig 2016.

\section{Results}

\section{Characteristics of the participating Centers}

A total of 64 (79\%) out of 81 departments participated in the survey with centers located in the Northeastern $(n=5)$, Northwestern $(n=11)$, Central Eastern $(n=6)$, Central Western $(n=23)$, and Southern states $(n=19)$ ( An almost equal distribution between university hospitals $(n=34)$ and non-university hospitals $(n=30)$ was achieved, respectively. Most of the participating institutions perform an annual load of 1,000 to 1,500 major cardiac operations (43.8\%), irrespective of the use of extracorporeal circulation and excluding cardiac rhythm device operations. The remaining departments are evenly distributed to the group of centers performing over 15,00 or $<1,000$ major procedures (28.1\% in each group). Thirty-two departments announced an annual volume of 200 to 400 operations on patients with acquired aortic valve diseases (including transcatheter aortic valve implantation [TAVI], but excluding pediatric patients with congenital heart disease), while eight

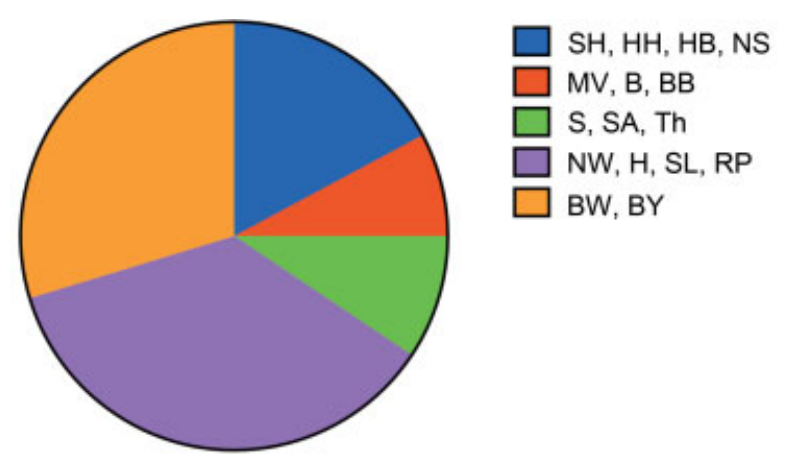

\section{Participating centers $(n=64)$}

Fig. 1 Regional distribution of cardiac surgical departments participating in the survey. B, Berlin; BB, Brandenburg; BW, Baden-Württemberg; BY, Bayern; H, Hessen; HB, Bremen; HH, Hamburg; MV, Mecklenburg-Vorpommern; NS, Niedersachsen; NW, NordrheinWestfalen; RP, Rheinland-Pfalz; S, Sachsen; SA, Sachsen-Anhalt; SH, Schleswig-Holstein; SL, Saarland; Th, Thüringen. 
departments had $<200$ aortic valve procedures performed per year, and 24 large-volume departments reported more than 400 aortic valve operations. Concerning specific operative techniques, it can be asserted that aortic valve replacements (AVR) using biological or mechanical prostheses were performed by all survey participants. Similarly, the majority of participating departments perform procedures involving aortic root replacement (95.3\%), TAVI (98.4\%), aortic valve sparing or reimplantation techniques (described by Yacoub or David; $96.9 \%)$, or isolated reconstruction of the aortic valve leaflets (85.9\%). In contrast, only $30 \%$ of all participating departments use a pulmonary autograft for AVR (Ross procedures).

In the majority of the institutions, an annual volume of 10 to $20(34.5 \%)$ operations or $>20(51.7 \%)$ operations for infective AVE was performed, while the total numbers of operations for prosthetic AVE was reported to be lower (5-10 operations in $40 \%$ and $>10$ operations in $37.9 \%$ ). More complex procedures involving AVR due to AVE as a concomitant procedure were less frequent (only 25 departments reported an annual case load $>10$ ).

\section{Perioperative Practice Related to Endocarditis Prevention and Follow-Up}

Among all participating departments, only 51 (80\%) enforce a dental work-up prior to elective operations on the aortic valve as a routine standard procedure. Perioperative antibiotic administration (endocarditis prophylaxis) is achieved by either first-generation cephalosporin (37.6\%) or secondgeneration cephalosporin (60.9\%), while in one institution, a combination of a second-generation cephalosporin and an aminoglycoside is favored. The respective antibiotics are most commonly administered 30 minutes prior to the estimated skin incision (67\%). A repeated administration after a certain duration of the operation is practiced by more than half of the participating centers (53.1\%). A smaller fraction of the participants prefer an administration at the time of skin incision (14\%) or at a time earlier than 30 minutes prior to skin incision (11\%).

In regards to discharge recommendations after an operation for infective endocarditis, a heterogeneous picture is revealed. We asked, whether immediate reconsultation is recommended to the patients and their referring physicians in case of fever, elevated inflammatory parameters, or abnormal echocardiographic findings. The latter recommendation is issued by $65.6 \%$ of the participating departments.

\section{Trends in the Intraoperative Management}

The choice of the optimal prosthesis for replacement of the infected aortic valve has been the focus of scientific investigation and a matter of long standing debate in the surgical community. Based on the results of this survey, stented xenografts were the prosthesis of choice for AVR in the instance of uncomplicated native AVE, i.e., without destruction of concomitant structures, as preferred by $65.5 \%$. In $22.4 \%$, neither heart valve prosthesis types is systematically favored, and in a minority of $8.6 \%$, a stentless xenograft is reported as the prosthesis of choice (-Fig. $\mathbf{2}$ ).

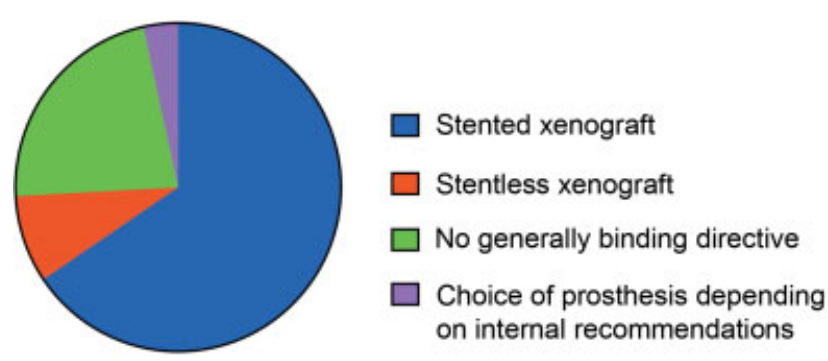

Fig. 2 Preferred prosthesis type for aortic valve replacement in the setting of infective endocarditis. Pie chart demonstrates the proportion of centers indicating respective prosthesis types as the overall preferred substitute for the infected aortic valve $(n=58)$.

In a more specific approach, the questionnaire asked for the cut-off age used to decide for a mechanical versus a biological heart valve prosthesis in infective AVE ( - Fig. 3). In seven departments, a mechanical heart valve prosthesis was not used in patients undergoing AVR for AVE, while in three departments, in all patients, a mechanical aortic valve prosthesis was chosen. In the remaining group of survey participants, a more liberal use of biological aortic valve prostheses becomes obvious, with almost one third choosing a biological prosthesis in patients at least 50 years old, and $>95 \%$ preferring a biological prosthesis for patients above 60 years of age. Although this survey had the specific background of infective endocarditis, its finding confirms a general trend to favor the use of biologic aortic valve prosthesis.

To elucidate the predominant surgical practice for the treatment of aortic root abscess formation, several common techniques were offered to choose from. In the majority of all responses, a technique using a pericardial patch to cover the abscess cavity was favored, whereas the implantation of a

\section{Preference of biological prosthesis}

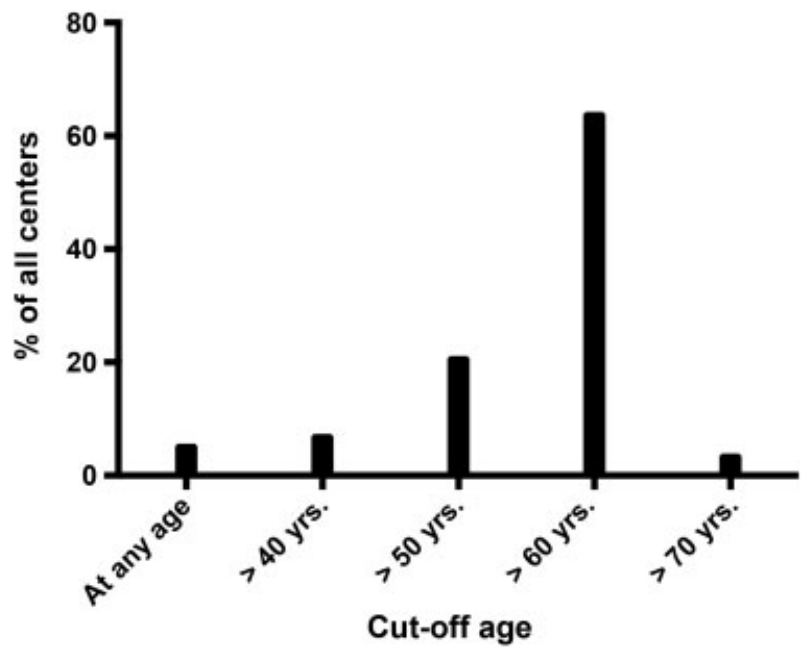

Fig. 3 Age cut-off for choice of biological prosthesis in the setting of IE. Proportion of departments that preferably use biological prostheses in the respective age cohorts of IE patients is indicated. IE, infective endocarditis; Yrs, years. 
biological conduit (18.9\%), a stentless xenograft (17.2\%) or a homograft (8.6\%) was less favorable, respectively. Finally a pericardial patch technique (5.1\%), draining the abscess into the pericardial cavity, or the implantation of a mechanical conduit (1.7\%) was least favored, respectively.

\section{Perioperative Diagnosis and Timing of Surgery}

Due to the significance of AVE-associated splenic abscesses, the preferred point of time for a splenectomy was explored. A vast majority of $84.5 \%$ indicated a staged procedure with splenectomy following valvular cardiac surgery as the standard protocol, whereas in only $15.5 \%$ splenectomy is performed prior to cardiac surgical AVR. In no case, a simultaneous operation was announced as the standard procedure, addressing the infected aortic valve and the splenic abscess.

According to the survey, a wide range of diagnostic steps are routinely performed prior to cardiac surgery for AVE. None of the offered multiple answer options, regarding the diagnostic steps were, chosen by any of the participants. The most frequently applied diagnostic examinations were transthoracic (98.1\%) and transesophageal echocardiography $(71.7 \%)$, cardiac catheterization for coronary angiography $(58.5 \%$; in $34 \%$, only in the absence of valvular vegetation), cerebral (60.4\%), thoracic (24.5\%) and abdominal computed tomography (CT) (37.7\%), and abdominal ultra-

\section{Preoperative examination}

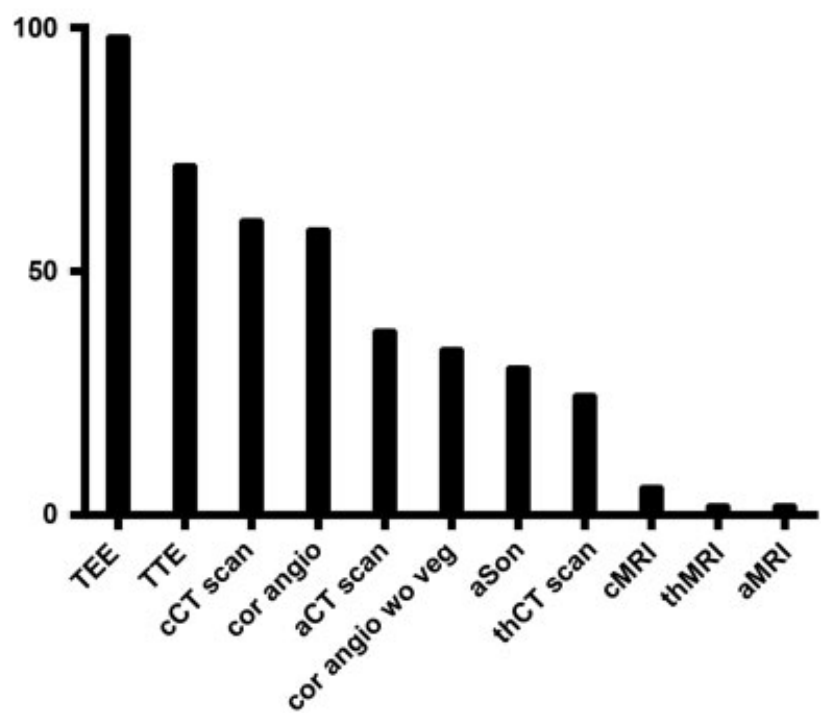

Fig. 4 Diagnostic tools routinely used for the planning of surgery for IE. Proportion of departments utilizing specific diagnostic methods as a routine tool in the perioperative treatment of patients with IE. The respective methods are in the order of their routine popularity as a routine step for patients undergoing cardiac surgery for IE. aCT scan, abdominal computer tomography scanning; aMRI, abdominal magnetic resonance imaging; aSon; abdominal sonography; cCT scan, cranial computer tomography scanning; cMRI, cranial magnetic resonance imaging; cor angio, coronary angiography; cor angio wo veg; coronary angiography performed only under the condition of absent vegetation; IE, infective endocarditis; TEE, transesophageal echocardiography; thCT scan, thoracic computer tomography scanning; thMRI, thoracic magnetic resonance imaging; TTE, transthoracic echocardiography. sonic examination (30.2\%). Magnetic resonance imaging (MRI) was less frequently applied (cerebral MRI in 5.7\%; thoracic or abdominal MRI in 1.9\%) ( - Fig. 4). A total of 79.3\% of the departments used the modified Duke criteria to confirm the diagnosis of IE, and the same number of participants considered the presence of a free mobile valvular vegetation, and its dimensions as a crucial factor for the indication for surgery. More specifically, vegetation dimensions greater than $10 \mathrm{~mm}$ were well accepted as an indication for surgery (66\%) and also lower dimensions in case of mobile vegetations ( $18.9 \%$ for $<5 \mathrm{~mm}$ and $30.2 \%$ for 5-10 mm).

Regarding the timing of cardiac surgery in the case of prior cerebral embolism, a wide range of strategies became evident (-Fig. 5). Answers for the optimal time interval varied between 24 hours and up to 6 weeks after the embolism, also included the option not to operate on these patients and the strategy of timing surgery irrespective to the event of cerebral embolism. The highest approval was noted for cardiac surgery under extracorporeal circulation (ECC) conditions irrespective of the time of cerebral embolism (36\%) or within the first 48 hours (30.2\%). In $24.5 \%$ of the participating departments, a preceding cerebral embolism with associated intracerebral bleeding was regarded as a contraindication for cardiac surgery, whereas $11.3 \%$ stated to perform surgery irrespective of the same finding.

\section{Diagnosis and Treatment of the Infectious Focus}

To gain an insight into the diagnostic testing and antibiotic treatment practice, the questionnaire asked for the rate of successful detection of the germs causing AVE. In slightly

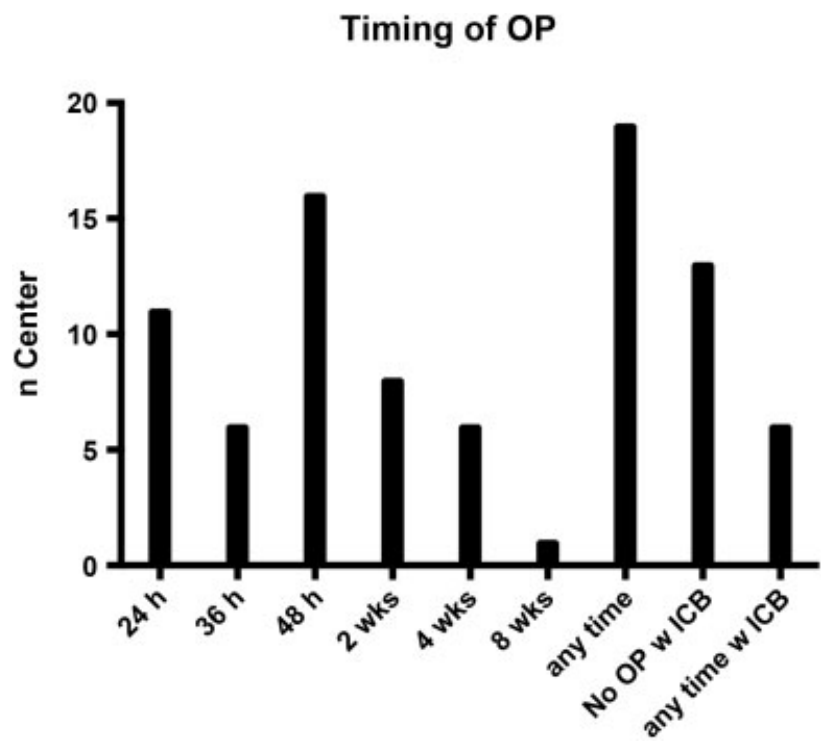

Fig. 5 Preferred timing of surgery for IE with cerebral embolization. Number of departments preferring cardiac operation for embolizing IE within specific time windows following a cerebral embolization. The two columns at the left indicate the number of departments that in general deny operative therapy in patients with cerebral embolization and intracranial bleeding (No OP w ICB) or those departments performing cardiac operation in the latter setting without delay (any time w ICB). h, hours; ICB, intracranial bleeding; IE, infective endocarditis; wks, weeks. 
more than half of all departments, a microbiological germ was identified as the cause of AVE in $>50 \%$ of the patients in the year 2014 , and in $32.1 \%$ of the participating departments, this rate exceeded $75 \%$ (-Fig. 6). The most frequently used probes allowing the detection of AVE were blood cultures (98\%), intraoperative swabs (92\%), microbiological cultures (94\%), and histology of valvular tissue material (90\%). Predominantly monoinfections were observed, $>75 \%$ of all patients in $54.7 \%$ of the participating sites and in 50 to $75 \%$ of all patients in $22.6 \%$ of all departments. Among the detected microorganism, fungal species was a rare entity with a proportion of $<1 \%$ in $66 \%$ of all participating departments. The most commonly reported species were Staphylococcus aureus (92.5\%), Staphylococcus epidermidis (77.4\%), unspecified Staphylococcus (83\%), unspecified Streptococcus (79.3\%), Escherichia coli (54.7\%), and Escherichia faecalis (45.3\%). Methicillin-resistant S. aureus (MRSA) and vancomycin resistant E. coli (VRE) were reported by $37.7 \%$ and $9.4 \%$ as the cause for IE in 2014, respectively ( - Fig. 7). There was a great variance regarding the frequency of cases in which the results of preoperative blood samples were subsequently confirmed by further microbiological evaluation of intraoperative samples (aortic valve tissue and other tissue samples). One fourth of all departments achieved such confirmation in $>75 \%$, while about one-fourth of the participants found such a confirmation in $<50 \%$ of their patients, and $28 \%$ of the participants reported to have no data on this issue.

A recurrent AVE (AVE in a patient with previous AVE in personal medical history) is less frequent and affects $<25 \%$ of the total number of AVE patients at the majority of the participating departments. However, we were not able to elucidate whether the recurrent AVE was predominantly caused by the same species as in the index AVE (58.5\% reported to have no data on this issue).

Besides the surgical therapy, antibiotic regimen remains a cornerstone in the treatment of AVE. According to our survey

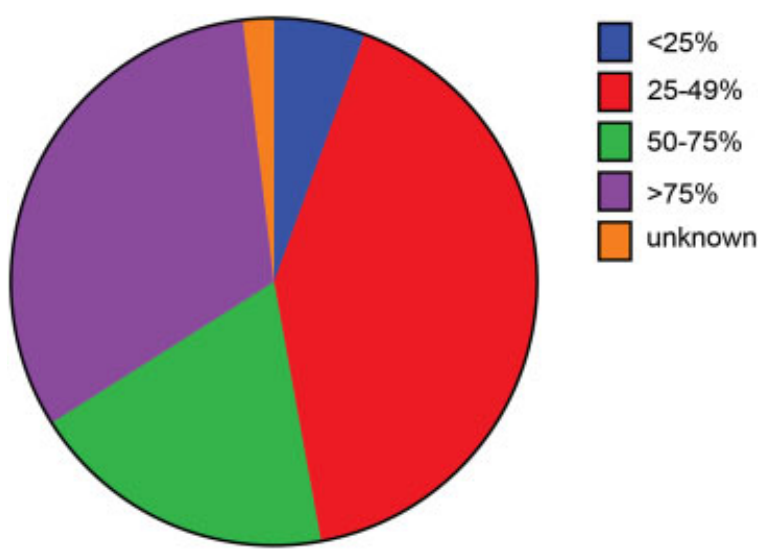

Fig. 6 Successful diagnostic confirmation of underlying organisms. The rate of successful diagnosis of the organism causing IE differs remarkably across the departments participating in the survey. In almost half of all departments, a successful diagnosis of the causative organism was performed in $>50 \%$ of all their IE patients, whereas $32 \%$ of all departments indicated a success rate above $75 \%$ (purple). IE, infective endocarditis.

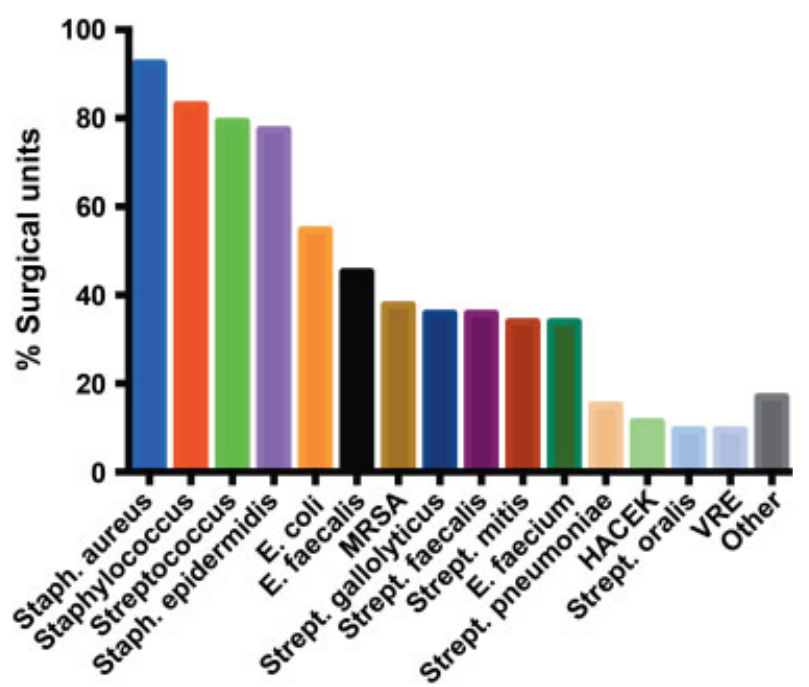

Fig. 7 Frequency of centers experience specific germs causing infective endocarditis of the aortic valve. Staphylococcus aureus and Streptococcus epidermidis were the most frequently reported subspecies and Staphylococci and Streptococci the most common bacterial families.

a calculated antibiotic therapy was most commonly performed with a combination of three antibiotic agents (54\%), while a smaller group applies a dual combination (16\%). Thirty percent of all responders indicated to treat their patients "according to the current guidelines," an option provided among the possible answers to this question. Following this topic, the specific antibiotics used for a calculated therapy were explored in a multiple choice manner. Gentamycin (60\%), vancomycin (56\%), and rifampicin (54\%) were the most frequently applied agents, while other antibiotics were selected by $30 \%$ or less of the participants. However, as for the previous question, the option "according to the current guideline" was frequently chosen (54\%). For recommending the duration of an antibiotic treatment, the day of operation was generally regarded as the relevant point in time marking the beginning of the time window (in $64 \%$ of participating departments), while in a smaller part of the participants, the preoperative begin was regarded as more relevant (16\%). In case of native AVE caused by S. aureus, the majority of the departments prefer an intravenous (IV) antibiotic treatment for 6 weeks following surgery, while in a minority of departments, IV treatment routinely exceeded 6 weeks (8\%) or remained shorter than 6 weeks ( 4 weeks in $24 \%$ and 2 weeks in $6 \%$ ). However, a trend toward longer IV antibiotic treatment was observed for prosthetic endocarditis with the same microorganism ( $\geq 6$ weeks in $86 \%$ of all departments). Finally, we asked for the standard treatment duration for Candida caused AVE. The most frequently applied duration of postoperative IV treatment was 6 weeks ( $44 \%$ ), while $42 \%$ preferred a prolonged treatment of $>6$ weeks. Similar to the trend observed for bacterial prosthetic valve AVE, the majority of $52 \%$ of departments prolonged IV treatment for $>6$ weeks in the event of prosthetic valve endocarditis. 


\section{Aortic Valve Endocarditis after Prosthetic Aortic Valve Replacement}

In the majority of the departments (62.3\%), a prosthetic heart valve endocarditis after AVR was observed $>1$ year after the initial operation. Interestingly, an early prosthetic AVE within the first year was more frequently observed when AVR was performed due to AVE: $52.8 \%$ of all participants described the first year after the index operation being the typical time frame for the occurrence of a recurrent AVE.

\section{Discussion}

The present work summarizes the results of a nationwide survey among cardiac surgical departments in Germany to determine the contemporary findings and practice in the surgical management of infective endocarditis. A total of 64 departments participated in the survey with an almost equal distribution between university hospital departments and departments outside of university hospitals. Considering the completeness of the data entry, the results of the current survey compare well with a previous nationwide survey initiated by the DGTHG and focusing on sternal wound complications after cardiac surgery. ${ }^{6}$ Moreover, the collected results from German cardiac surgical departments across all geographic regions of the country were collected, therefore providing the herein presented results with a representative character for the German-wide practice today, particularly when considering the overall landscape of cardiac surgical institutions in Germany and their annual performance. ${ }^{7}$

As a first crucial step in the management of AVE, the diagnostic process as practiced in the participating departments was explored. While TEE represented the standard diagnostic tool performed by almost all departments, neither a preoperative coronary angiography nor a CT scan was regarded as mandatory for all patients undergoing cardiac surgery for AVE. Indeed, class I recommendation for diagnosis (and surgical management of AVE) exists for TTE and TEE, whereas according to guidelines, cardiac CT scan is reasonable (class IIA, level of evidence B) when local complications, e.g., perivalvular abscess formation, are suspected. $^{3}$ In contrast, there is a lack of evidence for the benefit of coronary artery screening for patients undergoing cardiac surgery for AVE, and cardiac surgery without coronary angiography is a reasonable approach as recommended by the guidelines (class IIa) and frequently practiced in Germany according to the results of the presented survey. ${ }^{3}$

Most interestingly, a large diversity was observed regarding the indication and timing for cardiac surgery in case of septic embolization. As a matter of fact, large controversy continues on the optimal management of patients presented with recent stroke due to AVE-associated cerebral emboli. A recent report from a high-volume German department for cardiac surgery summarizing the results of 375 patients undergoing cardiac surgery for left heart IE with preoperative cerebral emboli underlines the severe impact of this complication. ${ }^{8}$ Preoperative cerebral, abdominal, and thor- acic CT scans were performed on all patients admitted with IE (a total of 1,571 patients). Based on the large cohort investigated, the authors demonstrated a similarly impaired outcome for both patients with clinically apparent cerebral emboli as well as patients with preoperative silent emboli detected with cranial CT scan or cranial MRI. The rate of postoperative hemiplegia as well as intracranial bleeding was comparable in both groups, despite the distinct preoperative clinical presentation of these cohorts. Although prospective data are yet missing, it appears likely that an intensified preoperative screening for extracardiac complications of IE may help to determine the specific treatment strategy in each individual patient. Data from other studies support the aforementioned results. A systematic screening by CT or MRI imaging has demonstrated cerebral mycotic aneurysms in $32 \%$ and abnormal findings in visceral organs in $34 \%$ of the analyzed patients. ${ }^{9}, 10$ Further reports based on MRI or CT findings estimate the proportion of IE patients suffering from a high rate of cerebral emboli, reaching up to $80 \%{ }^{11}$ Although not yet implemented in the current guidelines, a systematic neurological imaging prior to surgery for IE may improve the outcome and has been propagated by some authors. ${ }^{12} \mathrm{~A}$ second trend was an early operative therapy of IE even in case of silent or ischemic cerebral embolism. Although this strategy has to be validated by further prospective studies, this concept is already specified in the European and North American guidelines. ${ }^{2,13} \mathrm{~A}$ delayed operation is only recommended in cases of hemorrhagic stroke. ${ }^{2,13}$ The rationale behind this approach is derived from the fact that delayed surgery is more likely associated with systematic deterioration of the patient with less favorable overall outcome, while the benefit in terms of reduced risk of perioperative neurological exacerbation remains questionable. ${ }^{12}$

Another aspect of IE treatment as elucidated by the survey has to be further analyzed. Only half of all survey participants announced a positive finding of the underlying microorganism, which is significantly lower than rates previously published in Germany and elsewhere. ${ }^{8}$ However, as stated by the current European Society of Cardiology (ESC) guidelines, "positive blood cultures remain the cornerstone of diagnosis." It remains unclear whether the numbers reported by the survey participants reflect a true diagnostic gap currently present or whether other issues may have led to an artificially high rate of cases with missing diagnosis of the underlying organism.

Among the remarkable findings of this survey, a strong trend toward the use of xenografts for aortic valve replacement in the setting of AVE was noted, with a notable proportion of the departments generally excluding mechanical prosthesis for this indication. This trend is in line with the general tendency of a more liberal use of biological valve prostheses for heart valve replacement across Europe and worldwide, although data on the long-term outcome of young patients receiving biological prostheses is still inconsistent. $^{14}$

The results presented here are limited by the fact that not all of the cardiac surgical departments participated despite 
the repeated nationwide call by the DGTHG. Moreover, technical measures to ensure an anonymous modality of participation may have had an impact on the quality of data acquisition, e.g., the identity and organizational position of the person filling out the survey remains unclear. Finally, due to the voluntary nature of the survey as well as the aforementioned anonymous data supply, no structural mechanisms controlling the validity of the entered data were available. However, our results represent the largest data collection focused on the current practice in cardiac surgery for AVE and provide corner stones for planning and performing consecutive nationwide studies on AVE.

\section{Conclusion}

In summary, the results of the DGTHG survey on current practice for cardiac surgical therapy of AVE elucidate a heterogeneous picture with high uniformity of practice pattern in some aspects, e.g., high frequency use of TTE and TEE for preoperative diagnosis and planning. Controversial issues include the systematic screening for embolism as well as factors associated with preoperative diagnosis of the causative organism. These findings are a basis for a multicenter registry as a first step to improve knowledge on the nature and course of AVE across Germany. Moreover, a large multi-center registry will path the way for prospective trials addressing the most urgent clinical demand in the diagnosis and treatment of AVE.

\section{Funding}

The costs associated with design and distribution of the questionnaire as well as subsequent collection and analysis of the answers were covered by DGTHG and the institutional funds of the institutions of the authors of this work.

\section{Note}

This paper was presented at the 45th annual meeting of the German Society for Thoracic and Cardiovascular Surgery, Leipzig, February 2016.

\section{Conflict of Interest \\ None.}

\section{Acknowledgments}

The authors are grateful to all individuals and departments who have spent their time and effort in responding to the questionnaire and thereby making this report possible. The authors are particularly thankful for the support of board of the DGTHG as well as the DGTHG office team in managing the communication to the German cardiac surgical departments.

\section{References}

1 Nishimura RA, Otto CM, Bonow RO, et al. 2017 AHA/ACC focused update of the $2014 \mathrm{AHA} / \mathrm{ACC}$ guideline for the management of patients with valvular heart disease: a report of the American College of Cardiology/American Heart Association Task Force on
Clinical Practice Guidelines. J Am Coll Cardiol 2017;70(02): 252-289

2 Habib G, Lancellotti P, Antunes MJ, et al; ESC Scientific Document Group. 2015 ESC Guidelines for the management of infective endocarditis: the task force for the management of infective endocarditis of the European Society of Cardiology (ESC). Endorsed by: European Association for Cardio-Thoracic Surgery (EACTS), the European Association of Nuclear Medicine (EANM). Eur Heart J 2015;36(44):3075-3128

3 Nishimura RA, Otto CM, Bonow RO, et al; American College of Cardiology/American Heart Association Task Force on Practice Guidelines. 2014 AHA/ACC guideline for the management of patients with valvular heart disease: executive summary: a report of the American College of Cardiology/American Heart Association Task Force on Practice Guidelines. J Am Coll Cardiol 2014;63 (22):2438-2488

4 Nishimura RA, Carabello BA, Faxon DP, et al. ACC/AHA 2008 guideline update on valvular heart disease: focused update on infective endocarditis: a report of the American College of Cardiology/ American Heart Association Task Force on Practice Guidelines endorsed by the Society of Cardiovascular Anesthesiologists, Society for Cardiovascular Angiography and Interventions, and Society of Thoracic Surgeons. J Am Coll Cardiol 2008;52(08): 676-685

5 Delahaye F, Harbaoui B, Cart-Regal V, de Gevigney G. Recommendations on prophylaxis for infective endocarditis: dramatic changes over the past seven years. Arch Cardiovasc Dis 2009; 102(03):233-245

6 Beckmann A, Doebler K, Schaefer E, Koetting J, Gastmeier P, Graf K. Sternal surgical site infection prevention - is there any room for improvement? Eur J Cardiothorac Surg 2011;40(02): 347-351

7 Beckmann A, Funkat AK, Lewandowski J, et al. German Heart Surgery Report 2016: the Annual Updated Registry of the German Society for Thoracic and Cardiovascular Surgery. Thorac Cardiovasc Surg 2017;65(07):505-518

8 Misfeld M, Girrbach F, Etz CD, et al. Surgery for infective endocarditis complicated by cerebral embolism: a consecutive series of 375 patients. J Thorac Cardiovasc Surg 2014;147(06): 1837-1844

9 Iung B, Klein I, Mourvillier B, et al; Study Group. Respective effects of early cerebral and abdominal magnetic resonance imaging on clinical decisions in infective endocarditis. Eur Heart J Cardiovasc Imaging 2012;13(08):703-710

10 Meshaal MS, Kassem HH, Samir A, Zakaria A, Baghdady Y, Rizk HH. Impact of routine cerebral $C T$ angiography on treatment decisions in infective endocarditis. PLoS One 2015;10(03):e0118616

11 Cooper HA, Thompson EC, Laureno R, et al. Subclinical brain embolization in left-sided infective endocarditis: results from the evaluation by MRI of the brains of patients with left-sided intracardiac solid masses (EMBOLISM) pilot study. Circulation 2009;120(07):585-591

12 Yanagawa B, Pettersson GB, Habib G, et al. Surgical management of infective endocarditis complicated by embolic stroke: practical recommendations for clinicians. Circulation 2016;134(17): 1280-1292

13 Baddour LM, Wilson WR, Bayer AS, et al; American Heart Association Committee on Rheumatic Fever, Endocarditis, and Kawasaki Disease of the Council on Cardiovascular Disease in the Young, Council on Clinical Cardiology, Council on Cardiovascular Surgery and Anesthesia, and Stroke Council. Infective endocarditis in adults: diagnosis, antimicrobial therapy, and management of complications: a scientific statement for healthcare professionals from the American Heart Association. Circulation 2015;132(15):1435-1486

14 Zhao DF, Seco M, Wu JJ, et al. Mechanical versus bioprosthetic aortic valve replacement in middle-aged adults: a systematic review and meta-analysis. Ann Thorac Surg 2016;102(01):315-327 